\title{
Calificación laboral y desigualdad salarial: un ejercicio metodológico por conglomerados ${ }^{*}$
}

\author{
Labor Qualification and Wage Inequality: \\ a Methodological Exercise by Conglomerates
}

\author{
Albany Aguilera Fernández $z^{* *}$ David Castro Lugo ${ }^{* * *}$
}

\begin{abstract}
RESUMEN
En numerosos estudios relacionados con el análisis del mercado laboral, se ha requerido de emplear alguna clasificación de mano de obra de acuerdo con su grado de calificación; sin embargo, no existe un fundamento teórico que precise una alternativa para medir la calificación de los individuos. Tradicionalmente ésta se ha aproximado por años de estudio, nivel de escolaridad, ocupación que desarrolla o monto de las remuneraciones que percibe. El objetivo de este documento es presentar una propuesta metodológica basada en la técnica de conglomerados jerárquico aglomerativo que clasifica a la fuerza de trabajo en función de su nivel de calificación y, para ello, se toma como ejemplo el caso de México. Se consideran datos ocupacionales, nivel educativo y salario promedio para integrar tres categorías de trabajadores con características similares: calificados, semi calificados y no calificados. Los principales resultados confirman que el criterio seleccionado de clasificación de trabajadores influye en los valores de disparidad salarial.
\end{abstract}

Palabras clave: Calificación laboral, método clúster, desigualdad salarial, comercio.

Clasificación JEL: C14, F16, J21, J24, J31.

\begin{abstract}
In many studies related to the labor market analysis, it has been requested to have a classification according to skill level; however, there is no theoretical basis that provides an alternative to measure the workers skills. Traditionally, this is approximate by the education level, the employment that the worker develops or the labor remuneration. The main purpose of this study is to present a methodological exercise based on agglomerative hierarchical clustering techniques to classify the labor force according to qualification level. It is presented an example to the case of Mexico. In this case we considered occupational data, educational attainment and average wage to group together three labor categories with similar skill characteristics: skilled, semi-skilled and unskilled workers. The main results confirm that the criterion selected to classify workers influences the wage inequality values.
\end{abstract}

Keywords: Skill Classification, cluster method, wage inequality, trade.

JEL Classification: C14, F16, J21, J24, J31.

\footnotetext{
* Fecha de recepción: 23/05/2017. Fecha de aceptación: 24/05/2018. Los autores agradecen las observaciones y sugerencias de dos dictaminadores anónimos.

** Universidad Autónoma de Coahuila. Correo: albany.aguilera@uadec.edu.mx. ORCID: 0000-0001-5506-2901.

*** Universidad Autónoma de Coahuila. Correo: david.castro@uadec.edu.mx. ORCID: 0000-0003-0130-7197.
} 


\section{INTRODUCCIÓN}

La liberalización comercial como parte del proceso de globalización y el cambio tecnológico sesgado por calificación, se han propuesto como argumentos fundamentales para explicar la evolución de la demanda y de los salarios absolutos y relativos de la mano de obra (Castro y Huesca, 2007). De acuerdo con la teoría convencional del comercio internacional Heckscher-Ohlin y el teorema Stolper-Samuelson (SS), con la apertura al comercio los países en desarrollo deberán especializarse en la producción de bienes intensivos en mano de obra no calificada, lo que incrementará su demanda y remuneraciones relativas y, a su vez, reducirá el diferencial salarial entre éstos y los trabajadores más calificados (Stolper y Samuelson, 1941). En tanto que, la hipótesis de cambio tecnológico sesgado (HCTS) establece que el desarrollo de la tecnología no es neutral sobre la demanda de mano de obra, sustituye al trabajo no calificado y se complementa con el calificado; hecho que implicaría disminuir la demanda del primero y aumento del segundo, impactando sobre las remuneraciones y la desigualdad (Acemoglu y Autor, 2011; Rodríguez et al., 2011). De ahí que, en el contraste empírico de ambos enfoques, un elemento central lo constituye la determinación o clasificación de los trabajadores que integran los diferentes grupos según su calificación.

Reexaminando la literatura sobre el mercado laboral presente hasta el momento, no se ha encontrado una alternativa teórica para medir las calificaciones o, en estudios para México, un examen sistemático de clasificación de mano de obra. Empíricamente se han adoptado tres técnicas para aproximar el nivel de calificación: ocupaciones (Cañonero y Werner, 2002; Robertson, 2004; Airola y Juhn, 2005; Burgos y Mungaray, 2008; Acemoglu y Autor, 2011; Rodríguez et al., 2012), características educativas (Autor et al., 1998; Cortez, 2001; Aguayo-Téllez et al., 2010; Castro et al., 2013; Ríos y Carrillo, 2014), y en función de las remuneraciones (Leamer, 1996; Esquivel y Rodríguez-López, 2003). Aunado a lo anterior, la investigación en esta área ha optado por emplear clasificaciones previas o por introducir alguna según la información disponible, lo que conduce a establecer límites entre los distintos niveles de manera arbitraria y sin proporcionar un sustento al criterio seleccionado, pese a que cada uno tiene sus desventajas.

Dado lo cual, el objetivo del documento es presentar una propuesta metodológica con fundamento estadístico, para catalogar a los trabajadores según su nivel de calificación. Para ello se recurre al análisis de conglomerados jerárquico aglomerativo, técnica que permite conformar grupos homogéneos con respecto a las variables utilizadas para su formación y lo más distintos posible entre ellos. La finalidad es realizar una contribución al campo de la economía laboral al identificar de manera certera la estructura del mercado de trabajo, y superar la carencia observada de clasificaciones formales de mano de obra. 
En la aplicación del ejercicio metodológico se ejemplifica la experiencia de México, pero posee la ventaja de poder replicarse con los datos de cualquier otro país. Así, a partir de la base de 155 ocupaciones del Sistema Nacional de Clasificación de Ocupaciones (SINCO), e información sobre los años de escolaridad y el salario por hora promedio por ocupación, procedente de la Encuesta Nacional de Ocupación y Empleo (ENOE), se agruparon tres tipos de trabajadores: calificados, semi calificados y no calificados. Los resultados indican que la mano de obra calificada se concentra en ocupaciones que requieren mayor dotación de capital humano y reciben salarios más altos. Caso contrario se observó en la no calificada, que congrega a individuos que tienen hasta nueve años de escolaridad, correspondiente a una educación básica completa o menos. La categoría de semi calificados, conjunta labores con salarios y nivel educativo intermedios como coordinadores, auxiliares, técnicos, supervisores, entre otras.

A través de un comparativo de pruebas estadísticas y un análisis de disparidad salarial, se demuestra la pertinencia y aporte de la clasificación aquí propuesta, ya que se contrasta con alternativas de clasificaciones de mano obra utilizadas en la literatura previa. Adicionalmente se corrobora que el criterio de clasificación de trabajadores seleccionado influye sobre los valores de desigualdad de manera estadísticamente significativa.

El resto del documento se integra por cuatro apartados. En el primero se realiza una revisión de estudios previos que emplean algún criterio de desagregación de trabajo por tipo de calificación. Posteriormente se presenta la técnica de clasificación propuesta. En la tercera sección se efectúa un análisis de medidas de tendencia central, dispersión, y desigualdad salarial sobre un conjunto de clasificaciones de mano de obra, y finalmente las conclusiones.

\section{TRABAJO CALIFICADO Y NO CALIFICADO: UNA REVISIÓN DE DEFINICIONES}

En la literatura sobre el mercado laboral es común encontrar la distinción de dos clases de trabajadores: calificados y no calificados. Generalmente se acepta que el personal más calificado requiere destrezas que suponen un proceso de aprendizaje y experiencia, lo cual conlleva a una mejor remuneración. En tanto que, el menos calificado no precisa mayor instrucción ni conocimiento especial, lo compone cualquier individuo con educación básica y sus salarios suelen ser inferiores. No obstante, un problema que enfrentan los estudios que diferencian tipos de mano de obra, es seleccionar una variable óptima que aproxime la intensidad de calificación. 
De acuerdo con Leamer (1996), una adecuada forma de medir el empleo y los salarios implica encontrar subcategorías de trabajadores con un nivel de calificación uniforme dentro de los grupos, y sustancialmente diferente entre ellos. Para ciertos autores (Slaughter, 2000; Gonzaga et al., 2006; Winchester et al., 2006), las características educativas tienen una relación directa con las habilidades y aptitudes, por lo que resulta la medida más apropiada; mientras que otros se han postulado a favor de la división por ocupaciones (Tan y Batra, 1997; Betrán et al., 2007; Acemoglu y Autor, 2011; Rodríguez y Castro, 2012) para crear sustitutos de mano de obra calificada y no calificada.

\section{I.1. Clasificación de mano de obra por características educativas}

En relación con las características educativas, habitualmente se hace uso del nivel de educación o los años de escolaridad. Bhagwati y Dehejia (1993); Berman et al. (1994); Krueger (1997); Autor et al. (1998); Beyer et al. (1999); Gindling y Robbins (2001); Green et al. (2001); Francois y Nelson (2003) y Haskel et al. (2012) coinciden en establecer como umbral el nivel educativo y, en su mayoría, eligen la educación universitaria o superior para delimitar a los trabajadores calificados, y a los que cuentan con un nivel inferior los designan no calificados. Mientras que, en Cragg y Epelbaum (1996); Feliciano (2001); Airola y Juhn (2005); Aguayo-Téllez et al. (2010) y Castro et al. (2013) se conceptualizó como calificados a los individuos con al menos 12 años de escolaridad, y como no calificados a los que tienen estudios menores a 12 años.

Se ha inferido que si bien este criterio no garantiza el rendimiento laboral, refleja la capacidad para desempeñar trabajos de mayor responsabilidad y productividad, aunado a tener relación directa con las habilidades y aptitudes (Ramírez, 2004). Slaughter (2000) y Winchester et al. (2006) afirman que los datos educacionales son probablemente la medida idónea de las calificaciones, ya que ofrecen la ventaja de extenderse para incorporar varios tipos de mano de obra, lo que ha permitido que en algunos estudios integren un tercer grupo que han denominado semi calificados o de calificación media (Ramírez, 2000; Galiani y Sanguinetti, 2003; Terra y Patrón, 2010; Mishra y Kusum, 2013).

En contraste, aproximar las dotaciones de calificaciones adoptando únicamente las características educativas también presenta inconvenientes. En principio, no existe un consenso referente a la determinación de los distintos grupos, por lo que es frecuente que se establezcan límites exógenamente y sin fundamento, además de que con estos métodos sólo se consideran las calificaciones académicas (Winchester et al. (2006). Rodríguez y Castro (2012) señalan la limitante de asumir que los trabajadores se encuentran en ocupaciones que 
corresponden a su dotación de capital humano, conjetura que resultaría cuestionable en el caso del mercado laboral mexicano, por esta razón estiman que una categorización por ocupaciones define las habilidades, destrezas, capacidades u otro conjunto de herramientas que posee un individuo.

\section{I.2. Clasificación de mano de obra por ocupaciones}

Los enfoques de tipificación por ocupación generalmente utilizan la Clasificación Internacional Uniforme de Ocupaciones (CIUO), publicada por la Organización Internacional del Trabajo (OIT), como instrumento para clasificar los empleos y, en el caso de México, disponen de la Clasificación Mexicana de Ocupaciones (CMO) y el Catálogo Nacional de Ocupaciones (CNO). Bajo este esquema, relacionan al personal dedicado a labores que no implican producción como calificados, y a los de producción como no calificados (Revenga, 1997; Krueger, 1997; Cañonero y Werner, 2002; Robertson, 2004). Adicionalmente, se ha recurrido a las categorizaciones de trabajadores de cuello blanco/cuello azul y manual/no manual (Berman et al., 1994; Hanson y Harrison, 1995; Abrego y Whalley, 2002; Attanassio et al., 2004; Meza, 2003; Betrán et al., 2007), como indicativos de habilidades laborales.

El criterio ha sido justificado atendiendo a que, al emplear las encuestas oficiales de cada país, es la única definición de mano de obra que se tiene disponible (Abrego y Whalley, 2002; Meza, 2003). De acuerdo con Slaughter (2000), si bien la disociación producción/no producción es más propensa a clasificar erróneamente las calificaciones que una categorización basada en la educación, esto surge a raíz de problemas con los datos y no necesariamente de un sesgo.

\section{I.2.1. Clasificación de mano de obra desde} la perspectiva del cambio tecnológico

Desde otro enfoque para catalogar a la mano de obra, se ha relacionado la escolaridad y las ocupaciones de los individuos con la intensidad en el uso de las tecnologías. En este contexto, Autor et al. (2003) investigaron cómo la tecnología computacional alteró las demandas de calificaciones laborales; para ello definieron grupos por años de escolaridad, los cuales clasificaron a partir de las tareas que realizan: rutinarias y no rutinarias, y éstas en funciones analíticas e interactivas y manuales. Dicha técnica de clasificación fue base en análisis posteriores por Acemoglu y Autor (2011) para Estados Unidos y por Rodríguez et al. (2012) para el caso de México. Bajo este esquema, las tareas 
analíticas son las que retienen a los trabajadores más calificados por requerir mayor habilidad cognitiva, en tanto que las manuales suelen realizarse por individuos que tienen un menor nivel de instrucción.

Con el objetivo de observar el efecto que ha tenido la complementariedad de las tecnologías en el mercado laboral mexicano, Rodríguez et al. (2011), Rodríguez y Castro (2012) y Castro et al. (2013) delimitaron como mano de obra calificada a aquellos con 12 o más años de escolaridad terminada, y como no calificada a los que contaban con un nivel de estudios inferior a los 12 años. Además, fueron agrupados en ocupaciones de alta y baja intensidad tecnológica, a partir de la consideración de que la tecnología incrementa la productividad y los salarios del personal que realiza tareas que requieren su mayor utilización, a diferencia de aquellos que se ubican en actividades que implican un bajo o nulo contacto con las mismas.

Las principales objeciones por recurrir a procedimientos de clasificación por empleos subrayan las continuas transformaciones de los factores por el lado de la oferta, como el gradual aumento del nivel de instrucción de la fuerza de trabajo y el creciente capital humano, que potencialmente podrían generar variaciones en la composición de las tareas independientes de los cambios en la demanda. En paralelo, se ha demostrado que los trabajadores de cuello azul son mal clasificados cuando realizan actividades que demandan mayores destrezas; de igual manera, el nivel de educación no es adecuado cuando un individuo con alto grado de escolaridad ejecuta tareas que no requieren calificación (Gonzaga et al., 2006), de acuerdo con Delgado (2018), en México uno de cada tres trabajadores asalariados en el sector formal se encuentra sobreeducado. Adicionalmente, Wood (1997) reconoce que la división común cuello blanco (no producción) y cuello azul (producción) es poco confiable, ya que la composición de la calificación de ambos grupos es cambiante en el tiempo y, pese a que los datos por nivel de educación son más satisfactorios, no siempre están disponibles.

\section{I.3. Aproximación alternativa por salarios}

Leamer (1996) ha sido crítico del uso de ocupaciones como una medida para aproximar las calificaciones, por generarse agrupaciones amplias y heterogéneas que pueden conducir a catalogar erróneamente a demasiados trabajadores. Para superar las limitantes construyó una clasificación alternativa en la que asocia a los individuos que ganan salarios bajos con el trabajo no calificado, y a los que perciben salarios altos con el calificado. Si bien esta técnica es poco frecuente en relación con las aproximaciones por características educativas y ocupacionales, 
fue aplicada por Esquivel y Rodríguez-López (2003) y Garduño y Baylis (2012) en sus análisis para México.

Con lo discutido previamente se deduce que la literatura desarrollada hasta el momento no emplea un método confiable para la clasificación de mano de obra, puesto que la escolaridad y la ocupación de forma aislada no reflejan perfectamente la intensidad de calificación, ya que sólo pueden dar cuenta de manera inexacta de las diferencias en capacidades y habilidades de la población trabajadora. Atributos como la experiencia laboral, destrezas en comunicación, características individuales, capacidad de trabajar en equipo, el entorno institucional o la polarización de las condiciones de enseñanza entre escuelas públicas y privadas, pueden influir significativamente en los empleadores a la hora de seleccionar nuevo personal y en la determinación del ingreso (Ghiara y Zepeda, 2004; Salgado y Miranda, 2007). Por otra parte, la clasificación en función de las remuneraciones tiene el inconveniente de sufrir modificaciones asociadas a elementos coyunturales, que no necesariamente estarían evidenciando la naturaleza de las calificaciones de la mano de obra, inclusive factores de oferta y demanda pueden alterar los salarios relativos.

Ha de considerarse que la diversidad de aproximaciones al nivel de calificación de los trabajadores confirma que no hay un acuerdo general con relación a la definición de mano de obra calificada y no calificada. Lo que hasta ahora se ha presentado revela la multitud de criterios poco consistentes y, en su mayoría sin sustento, que se pueden encontrar en la literatura. Surge, por tanto, la necesidad de contar con un método robusto que establezca grupos lo suficientemente heterogéneos entre ellos, pero homogéneos en su interior, y determine lo mejor posible la calificación de los trabajadores.

\section{TÉCNICA DE CLASIFICACIÓN DE CALIFICACIÓN DE LA MANO DE OBRA}

Con el fin de identificar grupos con características similares de calificación, Winchester et al. (2006) proponen una técnica de clasificación en su estudio para el Reino Unido. Los autores integran calificaciones académicas, información de 77 ocupaciones provenientes de la Clasificación Ocupacional Uniforme (sOC por sus siglas en inglés) de 1990 y el salario medio, para aplicar una serie de técnicas clúster ${ }^{1}$ de agrupamiento jerárquico a los datos. De lo anterior delimitaron cuatro tipos de mano de obra: altamente calificados, calificados, semi calificados y no calificados.

1 A lo largo de este documento utilizaremos el término clúster como sinónimo de grupo, conglomerado y agrupamiento. 
Considerando lo innovador del método, y en virtud de que estudios previos han discriminado principalmente entre ocupaciones o características educativas definiendo grupos exógenamente y sin justificación, se decidió seguir el criterio de Winchester et al. (2006) y adaptar la técnica tomando como ejemplo el caso de México, con el objetivo de proporcionar una alternativa de clasificación certera y sustentada estadísticamente. Así, bajo la premisa de que los trabajadores calificados se encuentran en labores que requieren mayor habilidad, destreza y formación académica y, por consiguiente, perciben remuneraciones más altas en relación con los no calificados, el primer paso fue seleccionar las variables que describen y caracterizan la muestra.

\section{II.1. Datos}

Se recurrió al Sistema Nacional de Clasificación de Ocupaciones (SINCO), ${ }^{2}$ catálogo de clasificación estandarizado que permite homologar la información ocupacional con la que cuenta actualmente México. Para efectos de este documento se seleccionan 155 ocupaciones que corresponden al nivel de desagregación subgrupo. ${ }^{3}$

Adicionalmente, se incluyeron los años de escolaridad promedio de las ocupaciones y el salario por hora promedio de cada ocupación, con datos procedentes del tercer trimestre del 2012 de la Encuesta Nacional de Ocupación y Empleo (ENOE). ${ }^{4}$ Se dispuso del salario real por hora debido a que permite un examen más exacto, por lo que el salario mensual registrado en la ENOE a base 2012 se dividió por el producto de horas trabajadas por semana y $4.3 .^{5}$

\footnotetext{
El sINCO surgió como herramienta para sustituir las clasificaciones y catálogos ocupacionales que se habían utilizado en el país, como la Clasificación Mexicana de Ocupaciones (CMO) y el Catálogo Nacional de Ocupaciones (CNO).

3 El criterio de conformación de este nivel de agregación es jerárquico, combinado con algunas especializaciones como: nivel de responsabilidad, ámbito de acción de las ocupaciones, áreas o campos de conocimiento, especialización de los servicios prestados, modalidad de la venta, tipo de vigilancia y protección que se realiza, producto, tipo de material utilizado, secciones del proceso industrial y los materiales que se transforman, tratan, extraen, construyen o ensamblan, tipo de transporte, entre otros.

4 Corresponde la información más reciente disponible al momento de realizar la clasificación.

5 Número de semanas al mes.
} 


\section{II.2. Análisis de conglomerados jerárquico aglomerativo}

Partimos inicialmente de un análisis clúster conocido también como análisis de conglomerados, técnica estadística que presenta la ventaja de agrupar elementos que exhiben la máxima similitud interna y la mayor heterogeneidad externa. Por el contrario, los principales inconvenientes refieren que es un método no explicativo, y las soluciones dependen del procedimiento elegido y las variables incorporadas (De la Fuente, 2011).

Dentro de los algoritmos de formación de conglomerados se encuentra el método jerárquico aglomerativo, ${ }^{6}$ el cual consiste en una serie de técnicas que sitúan todos los casos en conjuntos homogéneos no conocidos previamente pero que los propios datos sugieren. En cada etapa del algoritmo se junta el par de grupos que maximice una medida de similitud o minimice la distancia, hasta llegar a un único clúster constituido por todos los elementos de la muestra (Vilá et al., 2014).

Si $n$ es el conjunto de casos de la muestra, de donde resulta el nivel $K=0$, con $n$ grupos, en el siguiente nivel se agruparán los dos casos que tengan la mayor similitud (o menor distancia), resultando así $n=1$ grupos; a continuación, se asociarán aquellos dos casos (o clúster ya formados) con menor distancia o mayor similitud; de manera que en el nivel $L$ se tendrá $n-L$ grupos establecidos. Así, hasta alcanzar el nivel $L=n-1$ en el que sólo hay un grupo compuesto por todos los casos de la muestra (De la Fuente, 2011).

Como procedimiento de agrupación jerárquica se optó por el método de Ward o de varianza mínima, que tiene como objetivo minimizar la varianza entre grupos. Fue propuesto por Ward (1963), quién dedujo que los conglomerados deberían constituirse de tal forma que, al unirse los elementos, la pérdida de información resultante de la fusión, medida a través de la suma de cuadrados del error ( $S C E$ ), fuese mínima. Por lo que en cada etapa se consideran todos los posibles pares de clúster, y se elige unir los dos cuya fusión produce el menor incremento en la $S C E$. Si el proceso comienza con $k$ conglomerados, cada uno compuesto por un solo individuo que coincide con el centro del clúster, en este primer paso se tendrá $S C E_{k}=0$ para cada clúster y con ello, $S C E=0$. El objetivo del método de Ward es encontrar en cada etapa dos agrupaciones cuya unión origine el menor aumento en la $S C E$. Así, el menor incremento de los errores cuadráticos es proporcional a la distancia euclídea al cuadrado de los centroides de los clúster unidos (Vilá et al., 2014; Pardo y Cabarcas, 2001; Winchester et al., 2006).

\footnotetext{
${ }^{6}$ En sentido inverso se encuentra el método jerárquico disociativo, el cual parte de un conglomerado conocido, que se divide en los siguientes pasos, formando grupos cada vez menores.
} 
Inicialmente $S C E_{k}=0, k=1, \ldots, N$ para los $N$ objetos. Si hay actualmente $K$ grupos, se define $S C E=\sum_{K=1}^{K} S C E_{k}$, donde

$$
S C E_{k}=\sum_{i=1}^{C_{K}} d^{2}\left(x_{i}, x_{k}^{\prime}\right)=\sum_{i=1}^{C_{K}}\left(x_{i}-x_{k}^{\prime}\right)^{t}\left(x_{i}-x_{k}^{\prime}\right)
$$

Donde $x_{k}$ es el centroide (media) y $C_{k}$ es el tamaño del clúster $k$.

La razón de adoptar este método se debe a varias ventajas: permite aplicar distintos tipos de medidas para estimar la distancia que existe entre variables, evita problemas de encadenamiento, minimiza la pérdida de información de cada agrupación de observaciones, es uno de los más utilizados en la práctica por maximizar la homogeneidad dentro de los grupos, suele ser discriminativo al determinar los niveles de agrupación, y tiende a crear clúster muy compactos de tamaño similar. Por el contrario, la principal desventaja refiere a que es sensible a los valores extremos, y no existe algún criterio general que permita establecer el número óptimo de conglomerados cuando se emplean técnicas clúster jerárquicas, la decisión es subjetiva (Redondo et al., 2003; De la Fuente, 2011).

Definido lo anterior, se inició con un examen preliminar de los datos para evaluar la posible influencia de casos atípicos, la existencia de correlación entre las variables y se estandarizaron las unidades a tratar. Como variable de proximidad entre los elementos, se seleccionó la distancia euclidiana al cuadrado ${ }^{7}$ y para aglomerar las ocupaciones se designó el método de Ward.

Se aplicaron dos técnicas para determinar el número de clúster que indique la estructura correcta y obtener una clasificación de los individuos lo más realista posible. En primer lugar, se analizó el dendograma, que constituye la representación gráfica en forma de árbol del resultado del procedimiento de unión, ${ }^{8}$ que es de significativa utilidad para evaluar la homogeneidad de los conglomerados. Adicionalmente, un método más formal consistió en exhibir gráficamente el número de clúster que se observan en los distintos pasos del algoritmo frente a la distancia a la que se produce cada fusión (anexo gráfica A1). Por consiguiente, la mejor solución de agrupar las 155 ocupaciones que pertenecen al subgrupo

Medida de disimilaridad que cuantifica el grado de diferencia o lejanía existente entre unidades. Tiene la ventaja de acelerar los cálculos, ya que no toma la raíz cuadrada. Recomendada en el algoritmo de Ward y en la que más influyen las diferencias en las medidas. Para $p$ variables se estima mediante la siguiente expresión (De la Fuente, 2011):

$$
d_{i j}^{2}=\sum_{K=1}^{p}\left(X_{i k}-X_{j k}\right)^{2}
$$

Donde $d_{i j}$ representa la distancia entre los casos $i$ y $j ; X_{i k}$ es el valor de la variable $X_{k}$ para el caso $i$; y $X_{j k}$ es el valor de la variable $X_{k}$ para el caso $j$.

8 Por cuestiones se espacio no se presenta en el documento el dendograma, no obstante, se encuentra disponible para quién lo solicite. 
del SINCO es la de tres conglomerados (anexo tabla A1), los cuales definimos como: 1) Trabajadores calificados, 2) Trabajadores semi calificados y 3) Trabajadores no calificados.

\section{II.3. Clasificación de mano de obra: interpretación de resultados}

Un análisis específico de las características de cada grupo de calificación ofreció más información sobre la naturaleza del agrupamiento, como se ilustra en el cuadro 1 y la gráfica 1. Es importante mencionar que, a partir de los valores de la desviación estándar, se observó menor grado de dispersión de los datos frente a otras clasificaciones efectuadas; por ejemplo, al incluir la variable del nivel de escolaridad promedio.

La gráfica 1 representa el diagrama de dispersión de los conglomerados de ocupaciones con respecto a las dos variables de categorización utilizadas: salario por hora y años de escolaridad promedio. Los casos están marcados según el grupo al que han sido asignados, es decir, en calificados, semi calificados y no calificados. La solución de tres conglomerados parece satisfactoria, ya que refleja la organización de los datos en las dos variables de clasificación. En resumen, estos resultados muestran que en general las ocupaciones que se identifican con trabajadores calificados tienen mayores ingresos salariales y nivel de escolaridad, caso contrario se distingue en las ocupaciones de menor calificación. En el anexo tabla A1, se describe detalladamente la asignación de las ocupaciones correspondientes a la clave del SINCO.

Cuadro 1. Clasificación de trabajadores por tipo de calificación.

\begin{tabular}{l|lrrr}
\hline \multicolumn{2}{c}{ Variables del análisis/Descriptivos } & \multicolumn{3}{c}{ Grupos } \\
\cline { 3 - 5 } \multicolumn{2}{c}{$\begin{array}{l}\text { Salario promedio } \\
\text { por hora }\end{array}$} & $\begin{array}{c}\text { Trabajadores } \\
\text { calificados }\end{array}$ & $\begin{array}{c}\text { Trabajadores } \\
\text { semi calificados }\end{array}$ & $\begin{array}{c}\text { Trabajadores } \\
\text { no calificados }\end{array}$ \\
\hline \multirow{2}{*}{$\begin{array}{l}\text { Años de escolaridad } \\
\text { promedio }\end{array}$} & Ocupaciones que integra & 71.92 & 42.18 & 24.17 \\
& Desviación estándar & 30 & 57 & 68 \\
\hline & Ocupaciones que integra & 13.02 & 10.83 & 5.58 \\
\hline
\end{tabular}

Fuente: Elaboración propia con datos del Instituto Nacional de Estadística y Geografía (INEGI). Encuesta Nacional de Ocupación y Empleo (ENOE), tercer trimestre del 2012. 
Gráfica 1. Dispersión de las ocupaciones según el nivel de calificación.

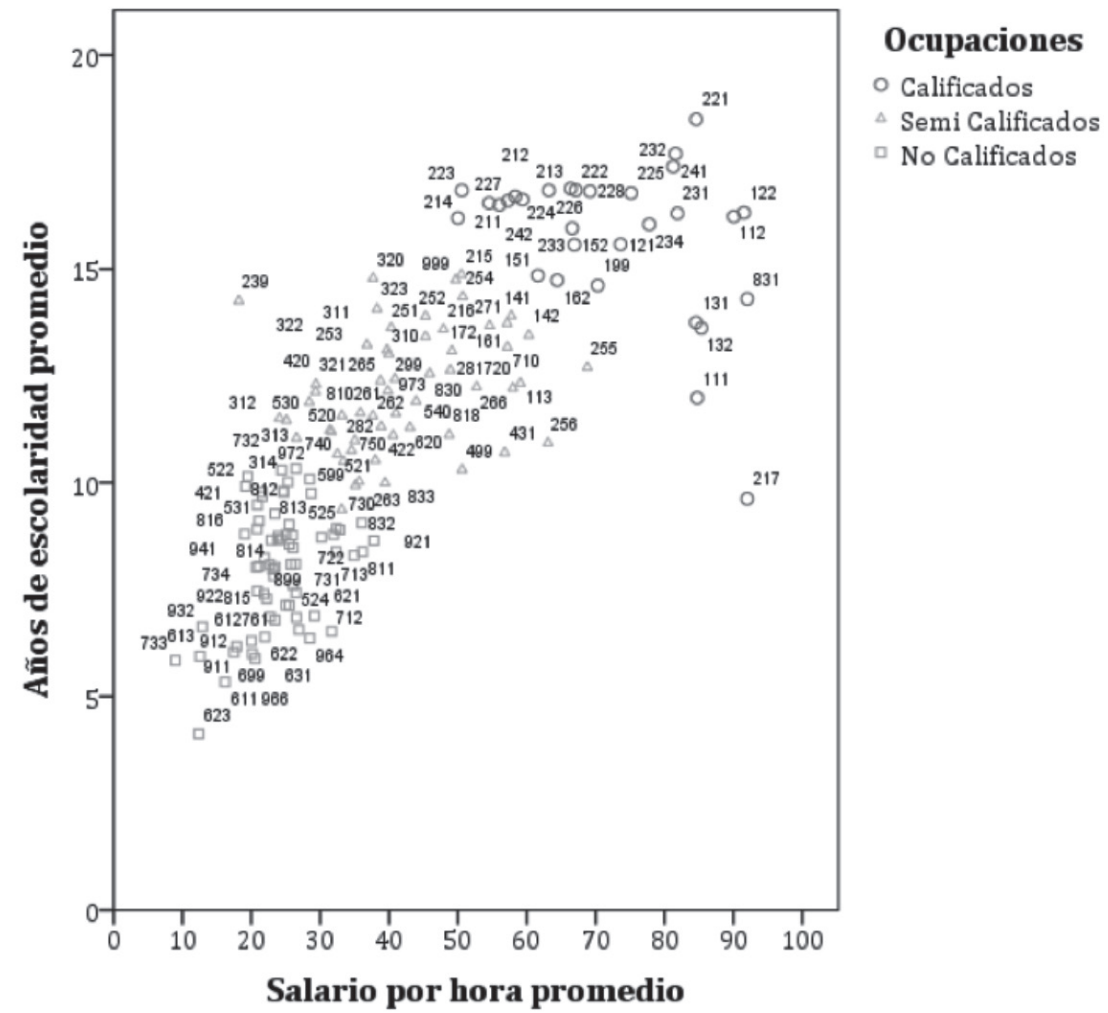

Nota: Pesos mexicanos de 2012. Tipo de cambio: pesos de México por dólares de Estados Unidos: 13.20.

Fuente: Elaboración propia con datos del Sistema Nacional de Clasificación de Ocupaciones (sINco) y de la Encuesta Nacional de Ocupación y Empleo (ENOE), tercer trimestre del 2012.

Los hallazgos revelan que los trabajadores calificados mexicanos se caracterizan por concentrarse en ocupaciones de directores y gerentes, coordinadores y jefes de área, administradores, investigadores, ingenieros, profesores, médicos, entre otros, que requieren mayor dotación de capital humano y son retribuidas con un salario más alto. Cabe señalar que, si bien un conjunto de documentos previos (Brauer y Hickok, 1995; Cragg y Epelbaum, 1996; Feliciano, 2001; Airola y Juhn, 2005; Borraz y López-Córdova, 2007; Huesca y Rodríguez, 2008; Aguayo-Téllez et al., 2010 y Castro et al., 2013) coinciden en definir como calificados a los que tienen al menos 12 años de escolaridad, los 
resultados aquí obtenidos sugieren que el promedio en México es superior a ese umbral, ya que actualmente estos cuentan con 15 o más años aproximadamente, equivalente a la conclusión del grado superior y estudios de posgrado (anexo tabla A1).

La categoría de semi calificados quedó conformada primordialmente por empleos de coordinadores, auxiliares, técnicos y supervisores (anexo tabla A1); con salarios y nivel educativo intermedios, correspondiente a una educación básica completa y superior incompleta (10 a 14 años de escolaridad). Lo anterior se asemeja a las definiciones ofrecidas por Galiani y Sanguinetti (2003) y Terra y Patrón (2010), aunque sus investigaciones son para Argentina y Uruguay, respectivamente.

En cuanto a los trabajadores mexicanos no calificados, se localizan principalmente en ocupaciones de artesanos, operadores, conductores de transporte, ventas, vigilancia, entre otros servicios, así como ciertas actividades relacionadas con el sector primario, lo cual es consistente con la especificación de Airola y Juhn (2005). Asimismo, perciben salarios inferiores, esto apoyaría la técnica empleada por Leamer (1996) y Esquivel y Rodríguez-López (2003), de asociar a los individuos que ganan salarios bajos con el trabajo no calificado y a los que reciben salarios altos con el calificado. Con respecto al nivel de escolaridad, resultó de hasta nueve años aproximadamente (anexo tabla A1), proporcional a una educación básica completa, y se ajusta al criterio de Ríos y Carrillo (2014).

Al comparar nuestros resultados con los obtenidos por Winchester et al. (2006), se distinguieron discrepancias. En primer lugar, difiere el periodo de análisis ya que ellos dispusieron de un catálogo de 1990 con un nivel de desagregación de 77 ocupaciones, así como seis posibles puntuaciones de la National Vocational Qualification (NVQ) para caracterizar el nivel educativo; y por medio del dendograma determinaron como número óptimo cuatro tipos de trabajadores (altamente calificados, calificados, semi calificados y no calificados). En el caso de México, con información más reciente se obtuvo una varianza mínima al conformar tres categorías (calificados, semi calificados y no calificados). Igualmente, se estima mayor homogeneidad intra y entre grupos al utilizar 155 ocupaciones y la variable años de escolaridad, lo cual contrasta el grado de calificación entre naciones desarrolladas y en desarrollo y, sugiere que esta clasificación podría utilizarse en aquellos países con una estructura educativa similar a México.

Así, consideramos que esta categorización cuenta con mayor solidez para reflejar la intensidad de calificaciones, dado que introduce una técnica sustentada estadísticamente para concentrar las variables fundamentales que definen la calificación de un trabajador. Es decir, contempla de manera conjunta, y no de forma individual como en la literatura precedente, características educativas, 
ocupacionales y salariales que, a su vez, incorporan elementos de instrucción formal, no formal (experiencia, capacitación, entre otras), así como capacidades innatas para obtener una agrupación más consistente. Representa una alternativa que tiene la cualidad de ser flexible, ya que permite aproximar el nivel de calificación ya sea por ocupaciones, grado educativo, años de escolaridad y/o salarios, lo que proporciona la ventaja de adaptarse a la información disponible en los estudios del mercado laboral.

\section{CALIFICACIÓN LABORAL Y DESIGUALDAD SALARIAL EN MÉXICO: UN CONTRASTE DE CLASIFICACIONES}

En aras de corroborar la relevancia y utilidad de la clasificación aquí propuesta, en relación con aquellas adoptadas en la literatura previa, en esta sección se presenta un comparativo de medidas de dispersión y desigualdad salarial. Se recurrió a la base de datos de la ENOE tercer trimestre del 2015, información más reciente disponible al momento de hacer el análisis; y para obtener la representatividad de la población se aplicó el factor de expansión que proporciona la encuesta. La muestra incluye a la población asalariada, tanto del sexo masculino como femenino que se encuentran en el rango de edad de entre 15 y 65 años.

Se inició estimando el coeficiente de variación para cada una de las definiciones de mano de obra que se exhiben en el cuadro 2, con el fin de examinar la dispersión relativa de los datos de ingreso. La primera columna corresponde a la clasificación expuesta en la sección anterior, a partir de la cual se identificaron tres tipos de trabajadores: calificados, semi calificados y no calificados. En la agrupación años de escolaridad, el personal calificado se constituye por los que tienen 12 o más años de escolaridad y los no calificados menos de 12 años (criterio utilizado en Brauer y Hickok, 1995; Cragg y Epelbaum, 1996; Feliciano, 2001; Airola y Juhn, 2005; Aguayo-Téllez et al., 2010 y Castro et al., 2013). La categorización grado educativo vincula con trabajadores calificados a los individuos que cuentan con educación universitaria o más, y no calificados con grado inferior a educación universitaria (alternativa empleada en Bhagwati y Dehejia, 1993; Berman et al., 1994; Krueger, 1997; Autor et al., 1998; y Beyer et al., 1999). La tipificación de tres categorías es la utilizada en Ramírez (2000), quien delimitó la mano de obra calificada si poseen estudios profesionales medios o superiores, semi calificada los que tienen más de seis años de primaria y hasta 12 años, y no calificada cuando reportan primaria terminada o menos.

En virtud de que nuestra clasificación sugerida es adaptable y, a su vez, permite aproximar el nivel de calificación con datos ocupacionales, se intentó realizar un comparativo con alguna categorización presentada en estudios 
precedentes, que haya empleado el criterio de ocupaciones. Sin embargo, se encontró la limitante que en ningún caso se señalan puntualmente la totalidad de empleos designados en cada categoría.

Cuadro 2. Coeficiente de variación del ingreso salarial en México por nivel de calificación, 2015.

\begin{tabular}{l|cccc}
\hline \multicolumn{1}{c}{ Trabajadores } & $\begin{array}{c}\text { Clasificación } \\
\text { propuesta }\end{array}$ & $\begin{array}{c}\text { Clasificación por } \\
\text { años de escolaridad }\end{array}$ & $\begin{array}{c}\text { Clasificación por } \\
\text { grado educativo }\end{array}$ & $\begin{array}{c}\text { Clasificación de } \\
\text { tres categorías }\end{array}$ \\
\cline { 1 - 2 } Calificados & $77.6 \%$ & $89.5 \%$ & $80.2 \%$ & $80.2 \%$ \\
\hline Semi calificados & $80.7 \%$ & na & na & $75.4 \%$ \\
\hline No calificados & $68.7 \%$ & $70.2 \%$ & $74.3 \%$ & $65.9 \%$ \\
\hline Total & $91.9 \%$ & $91.9 \%$ & $91.9 \%$ & $91.9 \%$ \\
\hline
\end{tabular}

Fuente: Elaboración propia con datos del Instituto Nacional de Estadística y Geografía (INEGI). Encuesta Nacional de Ocupación y Empleo (ENOE), tercer trimestre del 2015.

Los valores del coeficiente de variación que se exponen en el cuadro 2, confirman una mayor homogeneidad de la variable ingreso salarial para los conjuntos de trabajadores calificados ( 77.6 por ciento) y no calificados (68.7 por ciento) en la clasificación propuesta, en relación con los resultados obtenidos en las agrupaciones por años de escolaridad (89.5 por ciento y 70.2 por ciento) y por grado educativo (80.2 por ciento y 74.3 por ciento) que presentaron superior variabilidad. En la categorización de Ramírez (2000), la mano de obra calificada registró mayor dispersión de los datos (80.2 por ciento) que nuestro grupo, no siendo así para los restantes tipos de trabajadores.

Para evaluar la desigualdad del ingreso salarial se calculó el coeficiente de Gini (que varía entre 0 y 1 , siendo 1 el valor de mayor desigualdad). Cada ejercicio se replicó 100 veces a través del método bootstrap, con el fin de establecer intervalos de confianza del 95 por ciento en la estimación del indicador de desigualdad. Estos intervalos permiten evaluar si el cambio en las disparidades salariales, de acuerdo con el criterio de mano de obra seleccionado, fue estadísticamente significativo (ver cuadro 3). 
Cuadro 3. Desigualdad salarial en México según nivel de calificación, 2015 (Coeficiente de Gini).

\begin{tabular}{|c|c|c|c|c|c|c|c|}
\hline Trabajadores & $\begin{array}{l}\text { Clasificación } \\
\text { propuesta }\end{array}$ & $\begin{array}{l}\text { Clasificacic } \\
\text { años de escc }\end{array}$ & $\begin{array}{l}\text { ón por } \\
\text { olaridad }\end{array}$ & $\begin{array}{l}\text { Clasificacic } \\
\text { grado edu }\end{array}$ & $\begin{array}{l}\text { ón por } \\
\text { cativo }\end{array}$ & $\begin{array}{l}\text { Clasificaci } \\
\text { tres categ }\end{array}$ & $\begin{array}{l}\text { ión de } \\
\text { sorías }\end{array}$ \\
\hline \multirow{2}{*}{ Calificados } & 0.370 & & 0.400 & & 0.381 & & 0.381 \\
\hline & & {$[0.394$} & $0.406]$ & {$[0.375$} & $0.392]$ & {$[0.375$} & $0.387]$ \\
\hline \multirow{2}{*}{ Semi calificados } & 0.333 & & na & & na & & 0.304 \\
\hline & & & & & & [0 .299 & $0.309]$ \\
\hline \multirow{2}{*}{ No calificados } & 0.280 & & 0.286 & & 0.302 & & 0.275 \\
\hline & & {$[0.282$} & $0.291]$ & {$[0.297$} & $0.306]$ & {$[0.2693$} & $0.279]$ \\
\hline
\end{tabular}

Notas:

a) na indica que no aplica grupo de calificación en esa clasificación de mano de obra.

b) El ingreso corresponde al salario real por hora expresado en pesos del 2015.

c) Los valores en corchetes representan los intervalos de confianza al 95 por ciento.

d) Los valores en negrita y resaltados indican diferencia estadísticamente significativa.

Fuente: Elaboración propia con datos del Instituto Nacional de Estadística y Geografía (INEGI). Encuesta Nacional de Ocupación y Empleo (ENOE), tercer trimestre del 2015.

Pese a que la discrepancia en los valores del coeficiente de Gini obtenidos entre las distintas clasificaciones es reducida, las cifras que se muestran en el cuadro 3 reflejan una menor disparidad salarial al interior de los grupos de trabajadores calificados (0.37) y no calificados (0.28) en la clasificación propuesta, al compararlos con las categorías por años de escolaridad (0.40 y 0.29$)$ y grado educativo (0.38 y 0.30). En tanto que la población semi calificada registró mayor desigualdad (0.33) que la alcanzada en la agrupación de Ramírez (2000). No obstante, es importante señalar que, con base en los intervalos de confianza, en algunos casos las diferencias en el coeficiente de Gini resultaron estadísticamente significativas únicamente después de tres dígitos decimales.

El hecho de observar valores distintos al medir la distribución del ingreso, demuestra que el criterio de clasificación de trabajadores influye sobre los resultados de los índices de desigualdad, de ahí la relevancia de emplear una tipificación apropiada. Lo que se intentó con la técnica de agrupación presentada en este documento, es proporcionar una opción de clasificación de mano de obra elaborada a partir de un método estadístico formalizado, de manera que se conformen grupos lo más homogéneos en lo interno y heterogéneos en lo externo, permitiendo aproximar el nivel de calificación ya sea por ocupaciones, grado educativo, años de escolaridad y/o salarios, lo que ofrece una ventaja sobre aquellas alternativas que optan por un criterio y establecen límites exógenamente o sin sustento. 


\section{CONCLUSIONES}

A través de la revisión de literatura se comprobó que no hay un acuerdo general en lo que se refiere a la calificación de la mano de obra. Por tanto, advertimos la necesidad de contar con un método que proporcione un mayor sustento para estimar la intensidad de calificación. Dado lo cual, se tomó como ejemplo el caso de México para desarrollar una alternativa de categorización aplicando el análisis de conglomerados jerárquico aglomerativo, procedimiento que considera la similitud entre individuos para conformar grupos lo más homogéneos en su interior y heterogéneos entre ellos. Cabe recalcar que es posible aplicar esta metodología con los datos de otros países en desarrollo y desarrollados.

Mediante la técnica clúster se integraron tres elementos esenciales que describen la calificación de un trabajador: las ocupaciones que realiza, los años de escolaridad y el salario que percibe. A partir de lo anterior, se definieron tres grupos de trabajadores: calificados, semi calificados y no calificados. Los resultados indican que la mano de obra mexicana calificada se localiza en ocupaciones que requieren habilidades y destrezas, recibe salarios superiores y cuenta con más de 14 años de escolaridad, en tanto que el umbral de la no calificada fue de menos de nueve años, lo cual difiere de las categorías habituales que incluyen en el conjunto de calificados a los individuos con más de 12 años de educación y como menos calificados a los que tienen un nivel inferior. Los semi calificados se caracterizaron por tener entre 10 y 14 años de enseñanza formal y remuneraciones intermedias.

Si bien reconocemos que esta tipificación se mantendría para un determinado periodo de tiempo, ya que tanto los grados educativos de la población como los salarios ofrecidos en las distintas ocupaciones sufren continuas variaciones, consideramos que resulta una propuesta útil y certera para los estudios empíricos, a razón de que no se desagregan grupos injustificadamente, en su lugar se recurre a métodos estadísticos que facultan conformar agrupaciones con sujetos que muestran características simulares. Con respecto a otras clasificaciones existentes, tiene la ventaja de permitir aproximar la calificación de los individuos por ocupaciones, grado educativo, años de escolaridad y nivel de ingresos, ya sea en forma conjunta o individualmente.

Adicionalmente, la propuesta es capaz de reflejar la estructura del mercado laboral mexicano, dado que evidencia el aumento en el nivel de calificación de la población trabajadora, derivado de una acentuación en los años de escolaridad. Finalmente, se muestra conveniente cuando se requieran contrastar planteamientos teóricos que no especifiquen una alternativa para evaluar el nivel de calificación, como es el caso de la teoría convencional del comercio internacional. 


\section{REFERENCIAS BIBLIOGRÁFICAS}

Abrego, Lisandro y Whalley, John (2002), "Decomposing wage inequality change using general equilibrium models", National Bureau of Economic Research.

Acemoglu, Daron y Autor, David (2011), "Skills, tasks and technologies: Implications for employment and earnings", Handbook of Labor Economics, 4, pp. 1043-1171. ADDIN EN.REFLISTXA.

Aguayo-Tellez, Ernesto; Airola, Jim y Chinhui, Juhn (2010), "Did trade liberalization help women? The case of Mexico in the 1990s", National Bureau of Economic Research.

Airola, Jim y Chinhui, Juhn (2005), "Wage inequality in post-reform Mexico", IZA Discussion Paper, 48.

Attanasio, Orazio; Goldberg, Pinelopi y Pavcnik, Nina (2004), “Trade reforms and wage inequality in Colombia”, Journal of Development Economics, 74, pp. 331-366.

Autor, David; Katz, Lawrence y Krueger, Alan (1998), “Computing Inequality: Have Computers Changed the Labor Market?", The Quarterly Journal of Economics, 113 (4), pp. 1169-1213.

Autor, David; Levy, Frank y Murnane, Richard (2003), "The Skill Content of Recent Technological Change: An Empirical Exploration", The Quarterly Journal of Economics, 118 (4), pp. 1279-1333.

Berman, Eli; Bound, John y Griliches, Zvi (1994), "Changes in the demand for skilled labor within US manufacturing: evidence from the annual survey of manufacturers", The Quarterly Journal of Economics, 109 (2), pp. 367-397.

Betrán, Concha; Ferri, Javier y Pons, María (2007), "Wage Inequality and Globalisation: What Can We Learn from the Past? A General Equilibrium Approach", Documentos de Trabajo FUNCAS, 352.

Beyer, Harald; Rojas, Patricio y Vergara, Rodrigo (1999), “Trade liberalization and wage inequality", Journal of Development Economics, 59 (1), pp. 103-123.

Bhagwati, Jagdish y Dehejia, Vivek (1993), Freer trade and wages of the unskilled: is Marx striking again?, Columbia University, Department of Economics.

Borraz, Fernando y López-Córdova, José (2007), "Has Globalization Deepened Income Inequality in Mexico?", Global Economy Journal, 7 (1).

Brauer, David y Hickok, Susan (1995), "Explaining the growing inequality in wages across skill levels", Economic Policy Review, pp. 61-75.

Burgos, Benjamín y Mungaray, Alejandro (2008), "Apertura externa, inequidad salarial y calificación laboral en México, 1984-2002”, Problemas del Desarrollo, 39 (152), pp. 87-111.

Cañonero, Gustavo y Werner, Alejandro (2002), "Salarios relativos y liberación del comercio en México”, El Trimestre Económico, 69, pp. 123-142. 
Castro, David y Huesca, Luis (2007), “Desigualdad salarial en México: una revisión”, Papeles de Población, 13 (54), pp. 225-264.

Castro, David; Rodríguez, Reyna y Huesca, Luis (2013), "La calificación laboral en ocupaciones tecnológicas y no tecnológicas en México y sus regiones”, Estudios Sociales, 21 (42), pp. 87-112.

Cortez, Willy (2001), "What is Behind Increasing Wage Inequality in Mexico?", Word Development, 29 (11), pp. 1905-1922.

Cragg, Michael y Epelbaum, Mario (1996), "Why has wage dispersion grown in Mexico? Is it the incidence of reforms or the growing demand for skills?", Journal of development Economics, 51 (1) pp. 99-116.

De La Fuente, Santiago (2011), Análisis conglomerados, Universidad Autónoma de Madrid.

Delgado, Diego (2018), Desajuste educativo en un mercado laboral segmentado. El caso de México, 2005-2015, tesis doctoral, Doctorado en Ciencias Económicas, Universidad Autónoma Metropolitana-Iztapalapa, 147 pp.

Esquivel, Gerardo y Rodríguez-López, José (2003), “Technology, trade, and wage inequality in Mexico before and after NAFTA", Journal of Development Economics, 72 (2), pp. 543-565.

Feliciano, Zadia (2001), "Workers and trade liberalization: the impact of trade reforms in Mexico on wages and employment", ILR Review, 55 (1), pp. 95-115.

Francois, Joseph y Nelson, Douglas (2003), Globalization and relative wages: Some theory and evidence, University of Nottingham.

Galiani, Sebastian y Sanguinetti, Pablo (2003), "The impact of trade liberalization on wage inequality: evidence from Argentina", Journal of Development Economics, 72 (2), pp. 497-513.

Garduño, Rafael y Baylis, Kathy (2012), “Effect of Tariff Liberalization on Mexico's Income Distribution in the presence of Migration", Annual Meeting, agosto, pp. 12-14.

Ghiara, Ranjeeta y Zepeda, Eduardo (2004), "Desigualdad salarial, demanda de trabajo calificado y modernización: lecciones del caso de Tijuana, 1987-1994”, Región y Sociedad, 16 (29), pp. 4-43.

Gindling, Tim y Robbins, Donald (2001), "Patterns and sources of changing wage inequality in Chile and Costa Rica during structural adjustment”, World Development, 29, (4), pp. 725-745.

Gonzaga, Gustavo; Menezes, Filho y Terra, Cristina (2006), “Trade liberalization and the evolution of skill earnings differentials in Brazil", Journal of International Economics, 68, (2), pp. 345-367.

Green, Francis; Dickerson, Andy y Arbache, Jorge (2001), "A picture of wage inequality and the allocation of labor through a period of trade liberalization: the case of Brazil", World Development, 29 (11), pp. 1923-1939. 
Hanson, Gordon y Harrison, Ann (1995), "Trade, technology, and wage inequality", National Bureau of Economic Research.

Haskel, Jonathan; Lawrence, Robert; Leamer, Edward y Slaughter, Matthew (2012), "Globalization and U.S. wages. Modifying classic theory to explain recent facts", Journal of Economic Perspectives, 26, (2), pp. 119-140.

Huesca, Luis y Rodríguez, Reina (2008), "Salarios y calificación laboral en México", Problemas del Desarrollo, 39 (154), pp. 61-86.

Krueger, Alan (1997), "Labor market shifts and the price puzzle revisited", National Bureau of Economic Research.

Leamer, Edward (1996), "In search of Stolper-Samuelson effects on US wages", $\mathrm{Na}$ tional Bureau of Economic Research.

Meza, Liliana (2003), "Apertura comercial y cambio tecnológico: efectos en el mercado laboral mexicano", El Trimestre Económico, pp. 457-505.

Mishra, Prachi y Kusum, Deb (2013), "Trade liberalization and wage inequality in India: a mandated wage equation approach", Indian Growth and Development Review, 6 (1), pp. 113-127.

Pardo, Elias y Cabarcas, German (2001), "Métodos estadísticos multivariados en investigación social”, Simposio de Estadística, 13.

Ramírez, María (2000), "El empleo y la calificación de la mano de obra en México", Comercio Exterior, 50 (11). (2004), "Desigualdad salarial y desplazamientos de la demanda calificada en México, 1993-1999”, El Trimestre Económico, pp. 625-680.

Redondo, Carmen; Díez, José; Barreiro, Begoña y Freire, Miguel (2003), "La flexibilidad de los mercados de trabajo. Un análisis de las diferencias nacionales a través del trabajo a tiempo parcial", en Gestión científica empresarial: temas de investigación actuales, Netbiblo, pp. 141-158.

Revenga, Ana (1997), "Employment and wage effects of trade liberalization: the case of Mexican manufacturing", Journal of Labor Economics, 15 (S3).

Ríos, Jesús y Carrillo, Salvador (2014), "El empleo calificado y no calificado en la manufactura de México ante la crisis de 2009", Economía, Sociedad y Territorio, 14 (46), pp. 687-714.

Robertson, Raymond (2004), "Relative prices and wage inequality: evidence from Mexico", Journal of International Economics, 64 (2), pp. 387-409.

Rodríguez, Reyna y Castro, David (2012), "Efectos del cambio tecnológico en los mercados de trabajo regionales en México", Estudios Fronterizos, 13 (26) pp. 141-174.

Rodríguez, Reyna; Camberos, Mario y Castro, David (2012), “Cambio tecnológico y sustitución de trabajo en México y sus regiones”, Equilibrio Económico, 8 (1), pp. 31-66. 
Rodríguez, Reyna; Huesca, Luis y Camberos, Mario (2011), "Mercado laboral, inequidad salarial y cambio tecnológico regional”, Frontera Norte, 23 (45), pp. 7-33.

Salgado, María y Miranda, Sergio (2007), "Mercado de trabajo y profesionistas en el Estado de México", Quivera Revista de Estudios Territoriales, 9 (1), pp. 223-247.

Slaughter, Matthew (2000), "What are the results of product-price studies and what can we learn from their differences?", en The impact of international trade on wages, University of Chicago Press, pp. 129-169.

Stolper, Wolfgang y Samuelson, Paul (1941), "Protection and Real Wages", The Review of Economic Studies, 9 (1), pp. 58-73.

Tan, Hong y Batra, Geeta (1997), "Technology and firm size-wage differentials in Colombia, Mexico, and Taiwan (China)", The World Bank Economic Review, 11 (1), pp. 59-83.

Terra, María y Patrón, Rossana (2010), "Formación de capacidades en el Uruguay: ¿cuáles son las cualificaciones del trabajo requeridas para el desarrollo?”, en Comercio, pobreza y políticas complementarias en América Latina, Santiago, CEPAL, pp. 183-199.

Vilá, Ruth; Hurtado, María José; Berlanga, Vanessa y Fonseca, Mercé (2014), “Como aplicar un clúster jeràrquico en spss", REIRE. Revista d'Innovació i Recerca en Educació, 7 (1) pp. 113-127.

Ward, Joe (1963), "Hierarchical grouping to optimize an objective function", Journal of the American Statistical Association, 58 (301) pp. 236-244.

Winchester, Niven; Greenaway, David y Reed, Geoffrey (2006), "Skill classification and the effects of trade on wage inequality", Review of World Economics, 142 (2), pp. 287-306.

Wood, Adrian (1997), "Openness and wage inequality in developing countries: the Latin American challenge to East Asian conventional wisdom", The World Bank Economic Review, 11 (1), pp. 33-57. 


\section{Anexos}

Gráfica A1. Historial del proceso de conglomeración.

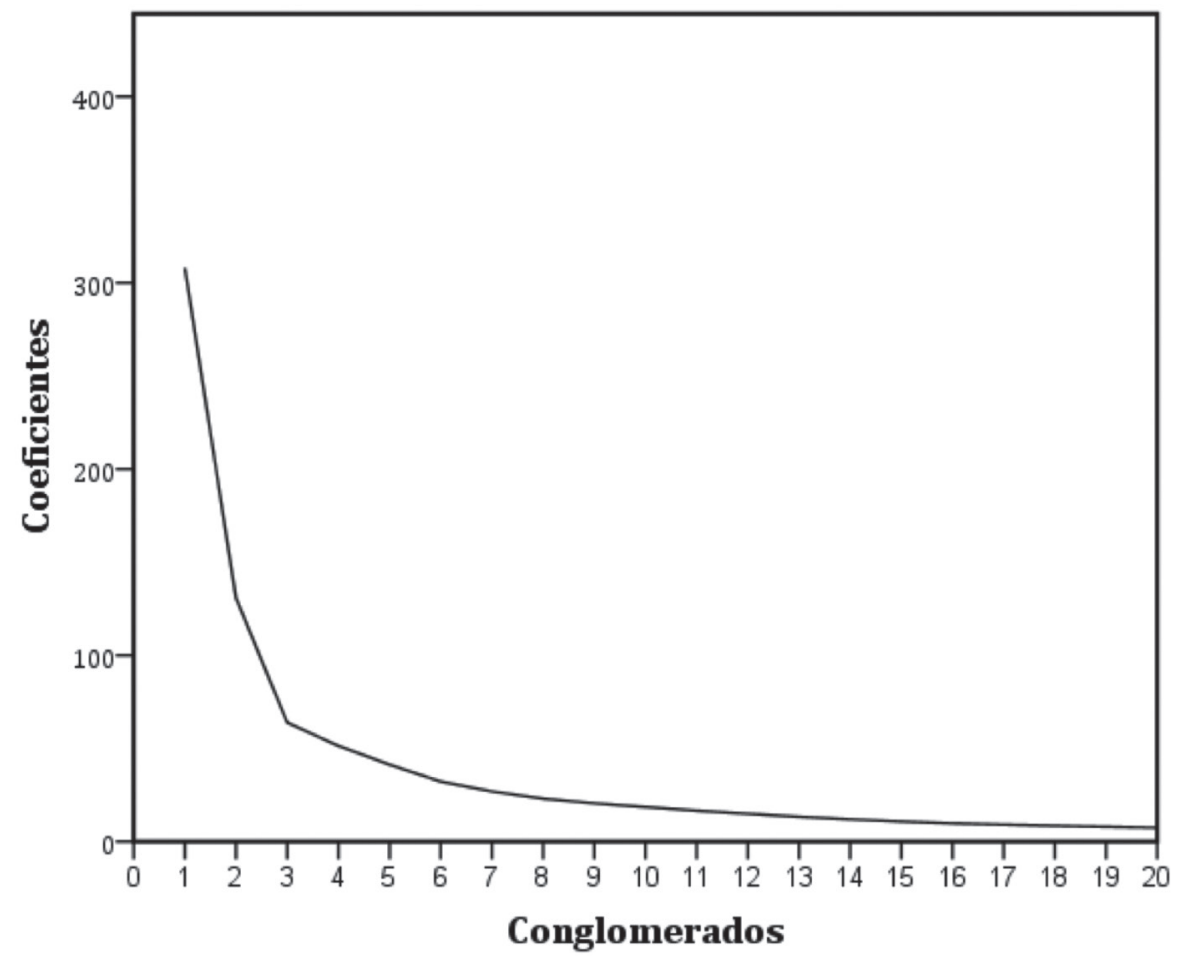

Fuente: Elaboración propia. 
Tabla A1. Clasificación de ocupaciones por tipo de calificación.

\begin{tabular}{|c|c|c|c|}
\hline $\begin{array}{l}\text { Clave } \\
\text { sinco }\end{array}$ & Ocupaciones & $\begin{array}{c}\text { Salario } \\
\text { por hora } \\
\text { promedio }\end{array}$ & $\begin{array}{l}\text { Años de } \\
\text { escolaridad } \\
\text { promedio }\end{array}$ \\
\hline \multicolumn{4}{|c|}{ Calificados } \\
\hline 111 & Funcionarios, legisladores y autoridades gubernamentales & 84.73 & 11.98 \\
\hline 112 & Presidentes y directores generales & 90.04 & 16.23 \\
\hline 121 & Directores y gerentes en servicios financieros y administrativos & 73.61 & 15.58 \\
\hline 122 & Directores y gerentes en servicios de salud, enseñanza y sociales & 91.52 & 16.32 \\
\hline 131 & $\begin{array}{l}\text { Directores y gerentes en producción agropecuaria, industrial, } \\
\text { construcción y mantenimiento }\end{array}$ & 84.56 & 13.75 \\
\hline 132 & $\begin{array}{l}\text { Directores y gerentes en informática, telecomunicaciones, transporte } \\
\text { y en investigación y desarrollo tecnológico }\end{array}$ & 85.34 & 13.62 \\
\hline 151 & Coordinadores y jefes de área en servicios financieros y administrativos & 61.61 & 14.85 \\
\hline 152 & $\begin{array}{l}\text { Coordinadores y jefes de área en servicios de salud, enseñanza, sociales } \\
\text { y jueces calificadores }\end{array}$ & 66.89 & 15.57 \\
\hline 162 & $\begin{array}{l}\text { Coordinadores y jefes de área en informática, telecomunicaciones, } \\
\text { transporte y en investigación y desarrollo tecnológico }\end{array}$ & 64.41 & 14.74 \\
\hline 199 & $\begin{array}{l}\text { Otros directores, funcionarios, gerentes, coordinadores y jefes de área, } \\
\text { no clasificados anteriormente }\end{array}$ & 70.30 & 14.62 \\
\hline 211 & Administradores y mercadólogos & 54.52 & 16.54 \\
\hline 212 & Contadores, auditores, especialistas en finanzas y en economía & 58.31 & 16.7 \\
\hline 213 & Investigadores y especialistas en ciencias sociales & 66.35 & 16.88 \\
\hline 214 & Investigadores y especialistas en ciencias humanistas & 50.00 & 16.19 \\
\hline 217 & Artistas interpretativos & 92.00 & 9.62 \\
\hline 221 & $\begin{array}{l}\text { Investigadores y especialistas en física, matemáticas, estadística } \\
\text { y actuaría }\end{array}$ & 84.60 & 18.5 \\
\hline 222 & $\begin{array}{l}\text { Investigadores y especialistas en ciencias biológicas, químicas } \\
\text { y del medio ambiente }\end{array}$ & 67.12 & 16.84 \\
\hline 223 & Especialistas en ciencias agronómicas & 50.56 & 16.84 \\
\hline 224 & Ingenieros eléctricos y en electrónica & 59.38 & 16.62 \\
\hline 225 & Ingenieros químicos, mecánicos, industriales, mineros y metalúrgicos & 75.13 & 16.78 \\
\hline 226 & Ingenieros civiles, topógrafos y arquitectos & 63.19 & 16.84 \\
\hline 227 & Investigadores y especialistas en sistemas computacionales & 57.27 & 16.59 \\
\hline 228 & Ingenieros en comunicaciones y telecomunicaciones & 69.18 & 16.82 \\
\hline 231 & Supervisores educativos y especialistas en ciencias de la educación & 81.86 & 16.3 \\
\hline 232 & Profesores de nivel medio y superior & 81.26 & 17.39 \\
\hline 233 & Profesores de nivel básico & 66.59 & 15.95 \\
\hline 234 & Profesores en enseñanza especial & 77.78 & 16.04 \\
\hline 241 & Médicos generales y especialistas & 81.58 & 17.69 \\
\hline 242 & Otros especialistas en salud & 56.03 & 16.5 \\
\hline 831 & Conductores de transporte aéreo & 92.00 & 14.3 \\
\hline \multicolumn{4}{|c|}{ Semi calificados } \\
\hline 113 & Directores de organizaciones políticas, sindicales y civiles & 59.06 & 12.33 \\
\hline 141 & Directores y gerentes de ventas, restaurantes y hoteles & 57.74 & 13.9 \\
\hline 142 & Directores y gerentes de museos, cines y otros establecimientos & 60.28 & 13.45 \\
\hline 161 & $\begin{array}{l}\text { Coordinadores y jefes de área en producción agropecuaria, industrial, } \\
\text { construcción y mantenimiento }\end{array}$ & 54.59 & 13.68 \\
\hline 171 & Coordinadores y jefes de área de ventas, restaurantes y hoteles & 45.29 & 13.42 \\
\hline 172 & Coordinadores y jefes de área en museos, cines y otros establecimientos & 49.11 & 13.08 \\
\hline
\end{tabular}


Tabla A1. Continuación.

\begin{tabular}{|c|c|c|c|}
\hline Slave & Ocupaciones & $\begin{array}{l}\text { Salario } \\
\text { por hora } \\
\text { promedio }\end{array}$ & $\begin{array}{l}\text { Años de } \\
\text { escolaridad } \\
\text { promedio }\end{array}$ \\
\hline 215 & Autores, periodistas y traductores & 50.52 & 14.87 \\
\hline 216 & Pintores, diseñadores y dibujantes artísticos, escultores y escenógrafos & 47.88 & 13.59 \\
\hline 239 & $\begin{array}{l}\text { Otros profesores y especialistas en docencia no clasificados } \\
\text { anteriormente }\end{array}$ & 18.17 & 14.25 \\
\hline 251 & Auxiliares en administración, contabilidad y finanzas & 40.29 & 13.63 \\
\hline 252 & Inspectores públicos & 45.29 & 13.89 \\
\hline 253 & Auxiliares en ciencias sociales y humanistas & 39.68 & 13.11 \\
\hline 254 & Diseñadores de modas, industriales, gráficos y decoradores de interiores & 50.71 & 14.35 \\
\hline 255 & Locutores, animadores y payasos & 68.78 & 12.7 \\
\hline 256 & Deportistas, entrenadores y árbitros & 63.07 & 10.93 \\
\hline 261 & $\begin{array}{l}\text { Auxiliares y técnicos en ciencias físicas, matemáticas, biológicas, } \\
\text { químicas, del medio ambiente y agronómicas }\end{array}$ & 39.80 & 12.15 \\
\hline 262 & $\begin{array}{l}\text { Auxiliares y técnicos industriales, topógrafos, mineros y dibujantes } \\
\text { técnicos }\end{array}$ & 37.64 & 11.57 \\
\hline 263 & $\begin{array}{l}\text { Mecánicos y técnicos en mantenimiento y reparación de equipo } \\
\text { mecánico, vehículos de motor, instrumentos industriales y equipos } \\
\text { de refrigeración }\end{array}$ & 35.11 & 9.93 \\
\hline 264 & $\begin{array}{l}\text { Técnicos eléctricos, en electrónica y de equipos en telecomunicaciones } \\
\text { y electromecánicos }\end{array}$ & 37.98 & 10.53 \\
\hline 265 & $\begin{array}{l}\text { Auxiliares y técnicos en informática y en equipos de comunicaciones } \\
\text { y grabación }\end{array}$ & 38.75 & 12.38 \\
\hline 266 & Controladores de tráfico aéreo y de otros transportes & 57.96 & 12.21 \\
\hline 271 & Auxiliares y técnicos en educación, instructores y capacitadores & 57.14 & 13.73 \\
\hline 281 & Enfermeras y técnicos en medicina & 48.89 & 12.63 \\
\hline 282 & Trabajadores de apoyo en salud & 38.85 & 11.32 \\
\hline 299 & Otros especialistas y técnicos, no clasificados anteriormente & 40.84 & 12.43 \\
\hline 310 & $\begin{array}{l}\text { Supervisores de secretarias, capturistas, cajeros y trabajadores de control } \\
\text { de archivo y transporte }\end{array}$ & 39.94 & 13.01 \\
\hline 311 & $\begin{array}{l}\text { Secretarias, taquígrafos, mecanógrafos, capturistas de datos } \\
\text { y operadores de máquinas de oficina }\end{array}$ & 36.79 & 13.23 \\
\hline 312 & Cajeros, cobradores y pagadores & 24.07 & 11.51 \\
\hline 313 & Trabajadores en archivo y control de almacén y bodega & 26.54 & 11.04 \\
\hline 320 & Supervisores de trabajadores que brindan y manejan información & 37.68 & 14.79 \\
\hline 321 & Recepcionistas, trabajadores que brindan información y telefonistas & 29.37 & 12.3 \\
\hline 322 & Trabajadores en agencias de viajes e información turística & 28.42 & 11.88 \\
\hline 323 & Encuestadores y codificadores & 38.24 & 14.07 \\
\hline 399 & $\begin{array}{l}\text { Otras secretarias, capturistas, cajeros y trabajadores que brindan } \\
\text { información, no clasificados anteriormente }\end{array}$ & 32.48 & 10.67 \\
\hline 420 & $\begin{array}{l}\text { Encargados y supervisores de ventas de productos y de servicios } \\
\text { financieros y de alquiler }\end{array}$ & 29.32 & 12.13 \\
\hline 422 & Agentes, representantes de ventas y vendedores por catálogo & 40.58 & 11.11 \\
\hline 423 & Trabajadores en la promoción de ventas y modelos & 31.60 & 11.2 \\
\hline 431 & Trabajadores en el alquiler & 56.78 & 10.7 \\
\hline 499 & $\begin{array}{l}\text { Otros comerciantes, empleados en ventas y agentes de ventas } \\
\text { en establecimientos, no clasificados anteriormente }\end{array}$ & 50.60 & 10.3 \\
\hline 510 & $\begin{array}{l}\text { Supervisores en la preparación y servicio de alimentos y bebidas, } \\
\text { así como en servicios de esparcimiento y de hotelería }\end{array}$ & 35.01 & 10.98 \\
\hline
\end{tabular}


Tabla A1. Continuación.

\begin{tabular}{|c|c|c|c|}
\hline $\begin{array}{l}\text { Clave } \\
\text { SInco }\end{array}$ & Ocupaciones & $\begin{array}{c}\text { Salario } \\
\text { por hora } \\
\text { promedio }\end{array}$ & $\begin{array}{c}\text { Años de } \\
\text { escolaridad } \\
\text { promedio }\end{array}$ \\
\hline 520 & $\begin{array}{l}\text { Supervisores y encargados de trabajadores en cuidados personales } \\
\text { y del hogar }\end{array}$ & 33.14 & 11.58 \\
\hline 521 & Peluqueros, embellecedores y similares & 35.64 & 10.03 \\
\hline 530 & Supervisores en servicios de protección y vigilancia & 25.09 & 11.46 \\
\hline 540 & Supervisores de la Armada, Ejército y Fuerza Aérea & 40.98 & 11.63 \\
\hline 620 & $\begin{array}{l}\text { Supervisores, encargados y capataces de trabajadores en actividades } \\
\text { pesqueras, acuícolas, forestales, caza y similares }\end{array}$ & 43.01 & 11.29 \\
\hline 710 & $\begin{array}{l}\text { Supervisores de trabajadores en la extracción, albañiles y en acabados } \\
\text { de la construcción }\end{array}$ & 57.16 & 13.18 \\
\hline 720 & $\begin{array}{l}\text { Supervisores de artesanos y trabajadores en el tratamiento y elaboración } \\
\text { de productos de metal }\end{array}$ & 52.70 & 12.24 \\
\hline 730 & $\begin{array}{l}\text { Supervisores de artesanos y trabajadores en la elaboración de productos } \\
\text { de madera, papel, textiles y de cuero y piel }\end{array}$ & 33.11 & 9.38 \\
\hline 740 & $\begin{array}{l}\text { Supervisores de artesanos y trabajadores en la elaboración de productos } \\
\text { de hule, caucho, plásticos y de sustancias químicas }\end{array}$ & 33.36 & 10.5 \\
\hline 750 & $\begin{array}{l}\text { Supervisores de trabajadores en la elaboración y procesamiento de } \\
\text { alimentos, bebidas y productos de tabaco }\end{array}$ & 34.53 & 10.76 \\
\hline 810 & Supervisores de operadores de maquinaria industrial & 35.83 & 11.64 \\
\hline 818 & Operadores de máquinas para la generación de energía & 48.72 & 11.12 \\
\hline 820 & $\begin{array}{l}\text { Supervisores en procesos de ensamblado y montaje de herramientas, } \\
\text { maquinaria, productos metálicos y electrónicos }\end{array}$ & 31.38 & 11.23 \\
\hline 830 & Supervisores de conductores de transporte y de maquinaria móvil & 45.89 & 12.55 \\
\hline 833 & Conductores de transporte en vías férreas & 39.41 & 10 \\
\hline 973 & Lecturistas de medidores, recolectores de dinero y elevadoristas & 43.92 & 11.9 \\
\hline 999 & Ocupaciones no especificadas & 49.67 & 14.75 \\
\hline \multicolumn{4}{|c|}{ No calificados } \\
\hline 314 & Trabajadores en el control de la operación de transporte & 24.70 & 9.78 \\
\hline 411 & Comerciantes en establecimientos & 26.05 & 8.48 \\
\hline 421 & Empleados de ventas y vendedores por teléfono & 19.47 & 10.14 \\
\hline 511 & $\begin{array}{l}\text { Trabajadores en la preparación y servicio de alimentos y bebidas } \\
\text { en establecimientos }\end{array}$ & 25.95 & 8.77 \\
\hline 522 & Trabajadores en el cuidado de personas & 19.16 & 9.91 \\
\hline 524 & Jardineros & 26.54 & 6.85 \\
\hline 525 & Trabajadores en otros servicios personales & 32.86 & 8.9 \\
\hline 531 & Trabajadores en servicios de protección y vigilancia & 21.11 & 9.1 \\
\hline 541 & Trabajadores de la Armada, Ejército y Fuerza Aérea & 28.47 & 10.09 \\
\hline 599 & $\begin{array}{l}\text { Otras ocupaciones en servicios personales y vigilancia, no clasificadas } \\
\text { anteriormente }\end{array}$ & 28.69 & 9.75 \\
\hline 610 & Supervisores, encargados y capataces agropecuarios & 25.76 & 8.09 \\
\hline 611 & Trabajadores en actividades agrícolas & 16.19 & 5.34 \\
\hline 612 & Trabajadores en actividades ganaderas y en la cría de animales & 20.01 & 6.3 \\
\hline 613 & Trabajadores que combinan actividades agrícolas con ganaderas & 12.55 & 5.94 \\
\hline 621 & Trabajadores en actividades pesqueras y de acuacultura & 29.17 & 6.88 \\
\hline 622 & Trabajadores en actividades silvícolas y forestales & 21.96 & 6.39 \\
\hline 623 & Trabajadores en actividades de caza, trampería y similares & 12.37 & 4.13 \\
\hline 631 & Operadores de maquinaria agropecuaria y forestal & 26.91 & 6.56 \\
\hline
\end{tabular}


Tabla A1. Continuación.

\begin{tabular}{|c|c|c|c|}
\hline $\begin{array}{l}\text { Clave } \\
\text { SINco }\end{array}$ & Ocupaciones & $\begin{array}{c}\text { Salario } \\
\text { por hora } \\
\text { promedio }\end{array}$ & $\begin{array}{c}\text { Años de } \\
\text { escolaridad } \\
\text { promedio }\end{array}$ \\
\hline 699 & $\begin{array}{l}\text { Otros trabajadores en actividades agrícolas, ganaderas, forestales, caza } \\
\text { y pesca no clasificados anteriormente }\end{array}$ & 20.12 & 6 \\
\hline 711 & Trabajadores en la extracción & 26.41 & 8.1 \\
\hline 712 & Albañiles y otros trabajadores en la edificación de construcciones & 31.66 & 6.52 \\
\hline 713 & $\begin{array}{l}\text { Yeseros, instaladores de pisos, climas, impermeabilizante, vidrio, etc., } \\
\text { plomeros y pintores }\end{array}$ & 34.89 & 8.3 \\
\hline 721 & Moldeadores, soldadores, hojalateros y pintores de metales & 32.39 & 8.93 \\
\hline 722 & $\begin{array}{l}\text { Herreros, cerrajeros, joyeros y artesanos en la elaboración de productos } \\
\text { de metal }\end{array}$ & 31.94 & 8.79 \\
\hline 731 & Artesanos y trabajadores en la elaboración de productos de madera & 26.48 & 7.42 \\
\hline 732 & $\begin{array}{l}\text { Artesanos y trabajadores en la elaboración de productos de papel, cartón } \\
\text { y trabajos de impresión }\end{array}$ & 24.39 & 10.3 \\
\hline 733 & Tejedores y trabajadores en la preparación de fibras textiles & 8.98 & 5.84 \\
\hline 734 & Artesanos y trabajadores en la elaboración de productos textiles & 20.89 & 7.47 \\
\hline 735 & $\begin{array}{l}\text { Tapiceros y trabajadores en el tratamiento y elaboración de productos } \\
\text { de cuero y piel }\end{array}$ & 25.51 & 7.13 \\
\hline 741 & $\begin{array}{l}\text { Artesanos y trabajadores en la elaboración de productos de hule, caucho, } \\
\text { plásticos y de sustancias químicas }\end{array}$ & 23.09 & 7.97 \\
\hline 751 & $\begin{array}{l}\text { Trabajadores en la elaboración y procesamiento de alimentos, bebidas } \\
\text { y productos de tabaco }\end{array}$ & 26.07 & 7.61 \\
\hline 760 & $\begin{array}{l}\text { Supervisores de artesanos y trabajadores en la elaboración de productos } \\
\text { de cerámica, vidrio, azulejo y similares }\end{array}$ & 23.93 & 8.75 \\
\hline 761 & $\begin{array}{l}\text { Artesanos y trabajadores en la elaboración de productos de cerámica, } \\
\text { vidrio, azulejo y similares }\end{array}$ & 23.44 & 6.79 \\
\hline 799 & Otros trabajadores artesanales, no clasificados anteriormente & 25.06 & 8.78 \\
\hline 811 & $\begin{array}{l}\text { Operadores de máquinas y equipos para la extracción en minas, } \\
\text { canteras y pozos }\end{array}$ & 36.15 & 8.38 \\
\hline 812 & $\begin{array}{l}\text { Operadores de máquinas y equipos en la fabricación metalúrgica, } \\
\text { fabricación de maquinaria y productos metálicos }\end{array}$ & 24.64 & 9.8 \\
\hline 813 & $\begin{array}{l}\text { Operadores de máquinas y equipos en la elaboración de productos } \\
\text { químicos, plásticos, tratamiento de agua y petroquímica }\end{array}$ & 23.41 & 9.27 \\
\hline 814 & Operadores de máquinas para la madera y papel & 22.88 & 8.66 \\
\hline 815 & $\begin{array}{l}\text { Operadores de máquinas y equipos en la elaboración de productos } \\
\text { textiles, cuero y piel }\end{array}$ & 20.72 & 8.03 \\
\hline 816 & $\begin{array}{l}\text { Operadores de máquinas en la elaboración de alimentos, bebidas } \\
\text { y tabaco }\end{array}$ & 20.89 & 9.48 \\
\hline 817 & $\begin{array}{l}\text { Operadores de máquinas en la elaboración de cemento y productos } \\
\text { de cerámica, vidrio y similares }\end{array}$ & 25.52 & 8.56 \\
\hline 819 & $\begin{array}{l}\text { Otros operadores de instalaciones y maquinaria fija industrial, } \\
\text { no clasificados anteriormente }\end{array}$ & 21.59 & 9.78 \\
\hline 821 & $\begin{array}{l}\text { Ensambladores y montadores de herramientas, maquinaria, productos } \\
\text { metálicos y electrónicos }\end{array}$ & 21.57 & 9.68 \\
\hline 832 & Conductores de transporte marítimo & 36.04 & 9.06 \\
\hline 834 & Conductores de transporte terrestre con motor & 25.49 & 9.02 \\
\hline 835 & Conductores de maquinaria móvil & 30.17 & 8.72 \\
\hline 899 & $\begin{array}{l}\text { Otros operadores de maquinaria industrial, ensambladores } \\
\text { y conductores de transporte no clasificados anteriormente }\end{array}$ & 22.24 & 7.29 \\
\hline
\end{tabular}


Tabla A1. Continuación.

\begin{tabular}{|c|c|c|c|}
\hline $\begin{array}{l}\text { Clave } \\
\text { SINCo }\end{array}$ & Ocupaciones & $\begin{array}{c}\text { Salario } \\
\text { por hora } \\
\text { promedio }\end{array}$ & $\begin{array}{c}\text { Años de } \\
\text { escolaridad } \\
\text { promedio }\end{array}$ \\
\hline 911 & Trabajadores de apoyo en actividades agropecuarias & 17.85 & 6.17 \\
\hline 912 & Trabajadores de apoyo en actividades forestales, pesca y caza & 17.46 & 6.03 \\
\hline 921 & Trabajadores de apoyo en la minería y extracción & 37.80 & 8.64 \\
\hline 922 & Trabajadores de apoyo en la construcción y la plomería & 21.83 & 7.4 \\
\hline 923 & Trabajadores de apoyo en la industria & 20.85 & 8.92 \\
\hline 931 & Ayudantes de conductores de transporte & 23.86 & 8.67 \\
\hline 932 & Conductores de transporte en bicicleta y animal & 12.87 & 6.63 \\
\hline 933 & Cargadores & 23.24 & 7.82 \\
\hline 941 & Ayudantes en la preparación de alimentos & 19.04 & 8.8 \\
\hline 951 & Vendedores ambulantes (excluyendo los de venta de alimentos) & 32.31 & 8.38 \\
\hline 952 & Vendedores ambulantes de alimentos & 25.00 & 7.13 \\
\hline 960 & $\begin{array}{l}\text { Supervisores en limpieza, amas de llaves, mayordomos } \\
\text { y en estacionamientos }\end{array}$ & 25.32 & 10.02 \\
\hline 961 & Trabajadores domésticos & 22.74 & 6.86 \\
\hline 962 & $\begin{array}{l}\text { Trabajadores de limpieza, recamaristas, mozos de limpieza y limpiadores } \\
\text { de calzado }\end{array}$ & 22.69 & 8.09 \\
\hline 963 & Lavadores y cuidadores de vehículos & 21.12 & 8.04 \\
\hline 964 & Lavanderos y planchadores & 28.49 & 6.36 \\
\hline 965 & Ayudantes de jardineros & 23.44 & 8.02 \\
\hline 966 & Recolectores de desechos, material reciclable y otros materiales & 20.57 & 5.89 \\
\hline 971 & Trabajadores de paquetería, empacado y de apoyo para espectáculos & 21.91 & 8.24 \\
\hline 972 & $\begin{array}{l}\text { Trabajadores repartidores de mensajería y mercancías } \\
\text { (a pie y en bicicleta) }\end{array}$ & 26.47 & 10.33 \\
\hline 989 & $\begin{array}{l}\text { Otros trabajadores en actividades elementales y de apoyo, } \\
\text { no clasificados anteriormente }\end{array}$ & 24.37 & 8.67 \\
\hline
\end{tabular}

Fuente: Elaboración propia con datos del Sistema Nacional de Clasificación de Ocupaciones (Sınco) y de la Encuesta Nacional de Ocupación y Empleo (ENOE), tercer trimestre del 2012. 
